\title{
Oxygenation-sensitive CMR for assessing vasodilator-induced changes of myocardial oxygenation
}

\author{
Matthias Vöhringer1,2, Jacqueline A Flewitt ${ }^{1}$, Jordin D Green 1 ,3, Rohan Dharmakumar ${ }^{4}$, Jiun Wang J J5 , John V Tyberg ${ }^{6}$ \\ and Matthias G Friedrich*7
}

\begin{abstract}
Background: As myocardial oxygenation may serve as a marker for ischemia and microvascular dysfunction, it could be clinically useful to have a non-invasive measure of changes in myocardial oxygenation. However, the impact of induced blood flow changes on oxygenation is not well understood. We used oxygenation-sensitive CMR to assess the relations between myocardial oxygenation and coronary sinus blood oxygen saturation $\left(\mathrm{SvO}_{2}\right)$ and coronary blood flow in a dog model in which hyperemia was induced by intracoronary administration of vasodilators.

Results: During administration of acetylcholine and adenosine, CMR signal intensity correlated linearly with simultaneously measured $\mathrm{SvO}_{2}\left(r^{2}=0.74, P<0.001\right)$. Both $\mathrm{SvO}_{2}$ and $\mathrm{CMR}$ signal intensity were exponentially related to coronary blood flow, with $\mathrm{SvO} 2$ approaching $87 \%$.

Conclusions: Myocardial oxygenation as assessed with oxygenation-sensitive CMR imaging is linearly related to $\mathrm{SvO}_{2}$ and is exponentially related to vasodilator-induced increases of blood flow. Oxygenation-sensitive CMR may be useful to assess ischemia and microvascular function in patients. Its clinical utility should be evaluated.
\end{abstract}

\section{Background}

Myocardial oxygenation, reflecting the balance or imbalance between oxygen demand and supply, is an important diagnostic target in various clinical settings[1], but may be especially useful for assessing ischemia and microvascular function. Presently available diagnostic tools are invasive, use exogenous contrast agents and/or radiation, are only useful in particular coronary territories, or have a limited spatial resolution[2,3]. Moreover, they do not provide direct measures of ischemia.

$\mathrm{T}_{2}{ }^{*}$-sensitive, Blood-Oxygen-Level-Dependent Cardiovascular MR (BOLD-CMR) uses the paramagnetic properties of deoxygenated hemoglobin as an endogenous contrast mechanism and is thus oxygenation-dependent[4]. In oxygenation-sensitive CMR images, the signal intensity of any soft tissue is inversely correlated with its

\footnotetext{
*Correspondence: matthias.friedrich@ucalgary.ca

7 Stephenson Cardiovascular MR Centre at the Libin Cardiovascular Institute of Alberta, Department of Cardiac Sciences, University of Calgary, Calgary, AB, Canada

Full list of author information is available at the end of the article
}

absolute content of deoxygenated hemoglobin and is therefore theoretically sensitive to changes in blood volume and oxygen supply-demand balance[5]. Such sequences have been used routinely for functional brain imaging[6] and similar approaches have been applied to the heart and peripheral perfusion beds [7-10]. Although these $\mathrm{T}_{2}{ }^{*}$ measurement and $\mathrm{T}_{2}{ }^{*}$-mapping techniques have been shown to have high BOLD sensitivity, they had limited clinical use thus far because of long acquisition times and relatively low signal-to-noise ratios. Additionally, magnetic field inhomogeneities, blood flow and cardiac motion may all impair image quality.

Recently, BOLD-sensitive, steady-state free precession (SSFP) techniques with much more consistent image quality have been introduced [11-13] and applied in experimental models of coronary artery stenosis[14,15]. To date, however, SSFP BOLD-CMR has not been validated against simultaneous measurements of myocardial oxygenation changes. Moreover, BOLD-weighted SSFP imaging has not been compared against other approaches such as $\mathrm{T}_{2}{ }^{*}$ mapping. 
We hypothesized that SSFP BOLD-CMR can accurately and consistently detect changes of myocardial oxygenation in vivo.

\section{Methods}

We used a canine model with selective intracoronary vasodilator infusion. In order to cover the full range of physiological flow changes we applied graded infusions of the endothelium-dependent vasodilator, acetylcholine, as well as the endothelium-independent vasodilator, adenosine.

\section{Animal Preparation}

Seven mongrel dogs (weight 15 to $25 \mathrm{~kg}$ ) were studied; all experiments were conducted in accordance with the most recent policies and "Guide to the Care and Use of Experimental Animals" by the Canadian Council on Animal Care. The local animal care and use board approved the study protocol and sample size.

Under general anesthesia, a midline sternotomy was performed and a 2-mm MR-compatible flow probe (Transonic Systems Inc., Ithaca, NY) placed around the proximal left circumflex (LCX) coronary artery. Under fluoroscopic control, a 2.7-F infusion catheter (Tracker18 Hi-Flow, Boston Scientific Ltd., Cork, Ireland) was introduced into the LCX through a diagnostic coronary catheter (JL 2.5, Torcon NB 'Advantage Catheter, Cook ', Denmark). The tip of the infusion catheter was placed a few millimeters proximal to the flow probe while ensuring that there were no visible side branches located between the infusion catheter and flow probe. In addition, a 4-F balloon catheter (Berman Angiographic Balloon Catheter, Arrow, Reading, PA, USA) was introduced into the coronary sinus (CS) for blood sampling. Blood gases were analyzed using a portable analyzer (STAT PROFILE ${ }^{\circ}$ Critical Care Xpress, Nova Biomedical, Waltham, MA, USA). All procedures including the CMR scan were performed in adjacent rooms with the dogs being placed on an MR-compatible cradle that allowed for a quick and easy transport to and from the MR system.

\section{CMR Protocol}

All CMR scans were performed in a clinical 1.5-T MRI system (MAGNETOM Avanto', Siemens Healthcare, Erlangen, Germany) with a 6-element phased-array coil resting on the chest and another below the spine. After acquiring localizer planes and performing manual regional shimming, BOLD-CMR was performed in a single mid-ventricular short-axis view at baseline (BL 1-3) and during intracoronary vasodilator infusion into the LCX. Acetylcholine (ACh) was infused in three increasing doses as previously described [16]: $0.1 \mu \mathrm{g} / \mathrm{min}$ (ACh 1), $1 \mu \mathrm{g} / \mathrm{min}$ (ACh 2) and $10 \mu \mathrm{g} / \mathrm{min}$ (ACh 3). Adenosine (Ade) was infused at the following rates: $30 \mu \mathrm{g} / \mathrm{min}$ (Ade
1), $150 \mu \mathrm{g} / \mathrm{min}$ (Ade 2) and $300 \mu \mathrm{g} / \mathrm{min}$ (Ade 3). Measurements were performed in the following sequence: $\mathrm{BL} 1$, ACh 1-3, BL 2, Ade 1-3, and BL 3. At the end of the protocol, we acquired a series of images during first-pass perfusion using a single-shot GRE-EPI sequence after intracoronary injection of $0.05 \mathrm{mmol} / \mathrm{kg}$ gadopentetate dimeglumine (Magnevist, Bayer, Germany) for accurately identifying the LCX perfusion territory and confirming the correct position of the intracoronary catheter.

SSFP BOLD-CMR was performed with a $\mathrm{T}_{2}{ }^{*}$-sensitive cine SSFP sequence as previously described [15]. Scan parameters were: $\mathrm{FOV}=228 \times 280 \mathrm{~mm}$; matrix size $=125$ $\times 192$; in-plane resolution $=1.8 \times 1.6 \mathrm{~mm}$; slice thickness $=5 \mathrm{~mm} ; \mathrm{TR} / \mathrm{TE}=5.8 \mathrm{~ms} / 2.9 \mathrm{~ms}$; flip angle $=90^{\circ}$; readout bandwidth $=275 \mathrm{~Hz} / \mathrm{Px}$; signal averages $=1$; the duration of the typical breath-hold was $15 \mathrm{~s}$. In addition, a segmented multi-echo gradient echo (GRE) sequence was used (echo train length: $8 ; \mathrm{T}_{\mathrm{E}}=2.6,4.8,7.0,9.3,11.5,13.7$, 16.0 , and $18.2 \mathrm{~ms}$ ) using a mono-polar readout. K-space lines from the different echoes were assigned to different images, and the resulting 8 images were used to generate a $\mathrm{T}_{2}{ }^{*}$ map. Other typical parameters for the multi-echo GRE sequence were: $F O V=225 \times 400 \mathrm{~mm}$; matrix size $=$ $109 \times 256$; in-plane resolution $=2.1 \times 1.6 \mathrm{~mm}$; slice thickness $=10 \mathrm{~mm}$; flip angle $=20^{\circ}$; TR $=120 \mathrm{~ms}$; and typical breath-hold duration, $16 \mathrm{~s}$. Reproducibility was assessed by 20 repeated baseline SSFP BOLD-CMR acquisitions in each of three subjects.

\section{Data Analysis}

CMR images were analyzed using certified software $\left(\mathrm{cmr}^{42}\right.$, CIRCLE Cardiovascular Imaging Inc., Calgary, Canada). A region of interest (ROI) was drawn around the LCX perfusion territory, which was clearly identified on the perfusion images as contrast was injected from the infusion catheter placed in the LCX (see Figure 1b). This ROI was then copied to all other images at the same slice location with careful adjustments to account for any mismatches. Myocardial signal intensity in SSFP BOLDCMR images and $\mathrm{T}_{2}{ }^{*}$ values were assessed for each protocol stage. The SSFP BOLD-CMR signal intensity was calculated for each of the 20 images obtained throughout the cardiac cycle and then averaged. $\mathrm{T}_{2}{ }^{*}$ maps were generated by fitting a mono-exponential function of $\mathrm{TE}$ through the $\mathrm{T}_{2}{ }^{*}$-weighted signal intensities.

Coronary blood flow was recorded from the Doppler flow probe $(\mathrm{ml} / \mathrm{min})$. Intra-arterial blood pressure was monitored continuously and the rate-pressure product (RPP) was calculated from heart rate and systolic pressure.

\section{Statistical Analysis}

Continuous variables with normal distribution were summarized as mean \pm standard error of the mean. Means at 


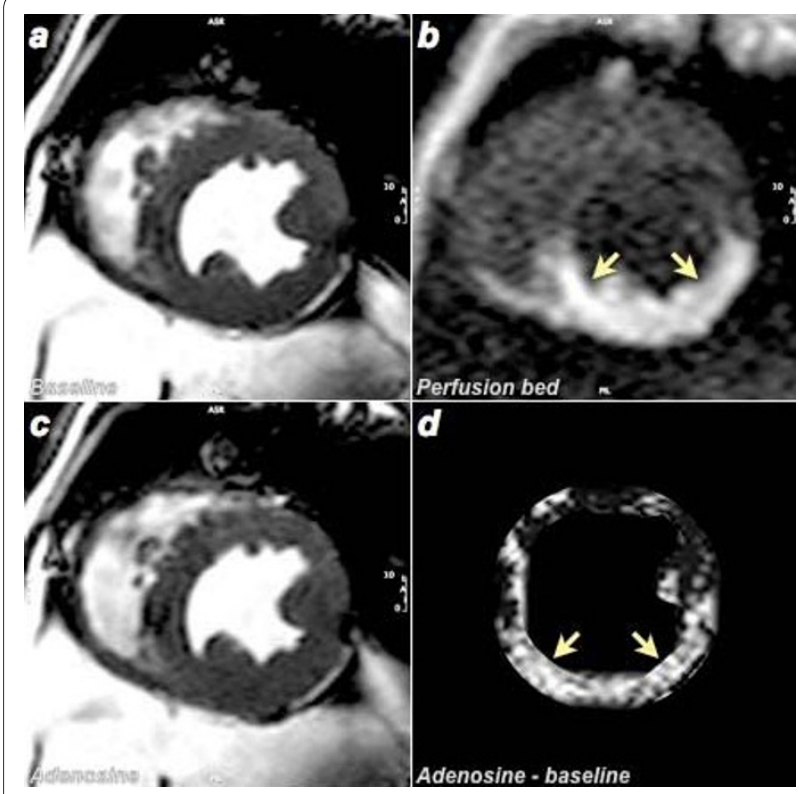

Figure 1 Examples of BOLD-CMR images a) at baseline; c) during infusion of $0.3 \mathrm{mg} / \mathrm{min}$ adenosine (Ade 3 ). The perfusion territory of the LCX after intracoronary injection of gadopentetate dimeglumine is shown in b) (arrows). Panel d) shows the myocardial signal intensity of baseline subtracted from that during adenosine. The higher values in the LCX territory reflect the signal intensity increase during in the LCX perfusion territory as induced by adenosine injection.

each level of drug infusion were analyzed with repeated measures ANOVA and post-hoc comparisons, using paired $t$-tests with Bonferroni adjustments (SPSS 16 for Macintosh, SPSS Inc, Chicago, USA). Correlation analysis was done (SIGMAPLOT, Windows version 10, Systat Software, Inc. San Jose, CA, USA) by linear regression analysis or by regression analysis using a 3-parameter, exponential-rise equation that asymptotically approached a maximum.

Sample-size calculation was performed aiming for the detection of a $200 \%$ increase in flow, which was considered to be relevant in assessment of myocardial perfusion reserve, with $80 \%$ power and $95 \%$ significance and based on results of previous studies with a similar experimental setting[11,17].

\section{Results}

Procedure

The drug-infusion protocol was completed and the perfusion territory of the LCX was identified in all 7 animals (examples see Figure $1 \mathrm{~b}$ and $2 \mathrm{~d}$ ). In three animals, the adenosine perfusion protocol was shortened because of difficulties during the surgical or interventional procedure. One of the protocol stages in two of these three animals and two protocol stages in the third could not be performed. In one dog, the coronary sinus catheter dislocated after the baseline study and could not be relocated,

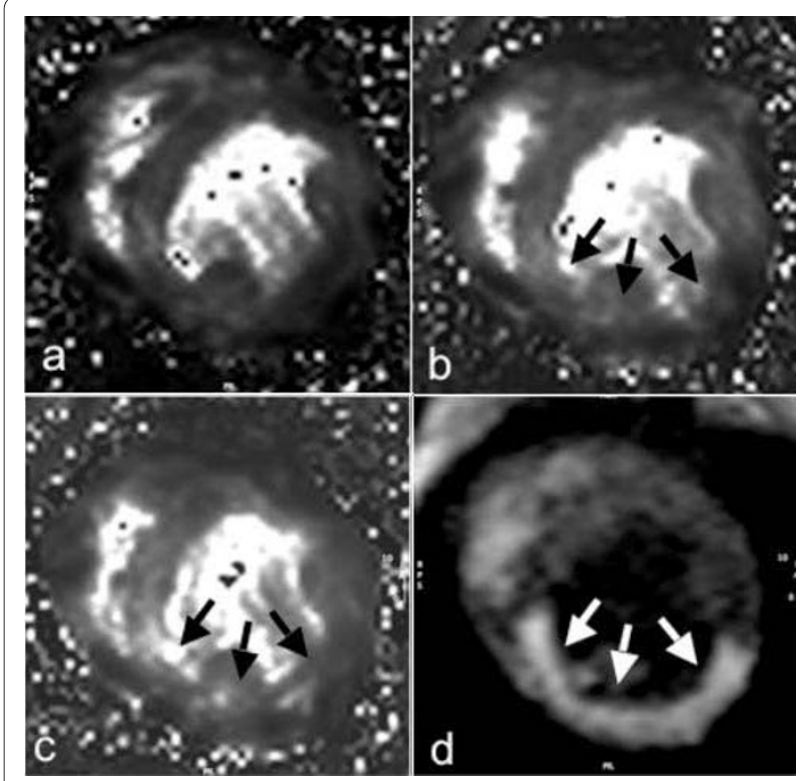

Figure 2 Examples of $\mathrm{T}_{2}{ }^{*}$ maps at a) baseline, b) during $\mathrm{LCX}$ infusion of $10 \mu \mathrm{g} / \mathrm{min}$ acetylcholine (ACh 3 ) and c) during infusion of $0.3 \mathrm{mg} / \mathrm{min}$ adenosine (Ade 3 ). The perfusion territory of the LCX after intracoronary injection of gadolinium is shown in d) and indicated with white arrows.

so blood gas analysis from the coronary sinus was not possible. In another dog, a different SSFP BOLD-CMR sequence was used and only $\mathrm{T}_{2}{ }^{*}$ data was included in the analysis.

\section{Coronary Vasodilation Experiments}

Throughout the experiments, there were no significant changes in heart rate, blood pressure and RPP when compared to baseline (Table 1). Drug-induced vasodilation significantly increased LCX blood flow, whereas there was no significant difference between the two repeated baseline measurements (Table 1). There was a dosedependent increase in blood flow, which was accompanied by progressively smaller increases in coronary sinus oxygen saturation $\left(\mathrm{SvO}_{2}\right)$. Using the 3-parameter, exponential-rise equation, $\mathrm{SvO}_{2}$ correlated with $\mathrm{LCX}$ blood flow $\left(\mathrm{r}^{2}=0.84, P<0.001\right)$ with $\mathrm{SvO}_{2}$ approaching $87 \%$ (Figure 3). Mean arterial oxygen saturation was $96.7 \pm$ $1.2 \%$.

\section{Oxygenation-sensitive CMR}

SSFP BOLD-CMR consistently provided very good image quality (Figure 1). Although image quality of $\mathrm{T}_{2}$ * maps was consistent, the images showed distortion and susceptibility artifacts, especially with respect to the infero-lateral wall (Figure 2). Representative images for baseline, high-dose ACh and/or high-dose Ade are shown in Figures $1 \mathrm{a}, \mathrm{c}$ and $2 \mathrm{a}, \mathrm{b}$ and $2 \mathrm{c}$. After subtracting baseline image signal intensity, changes of signal intensity were 


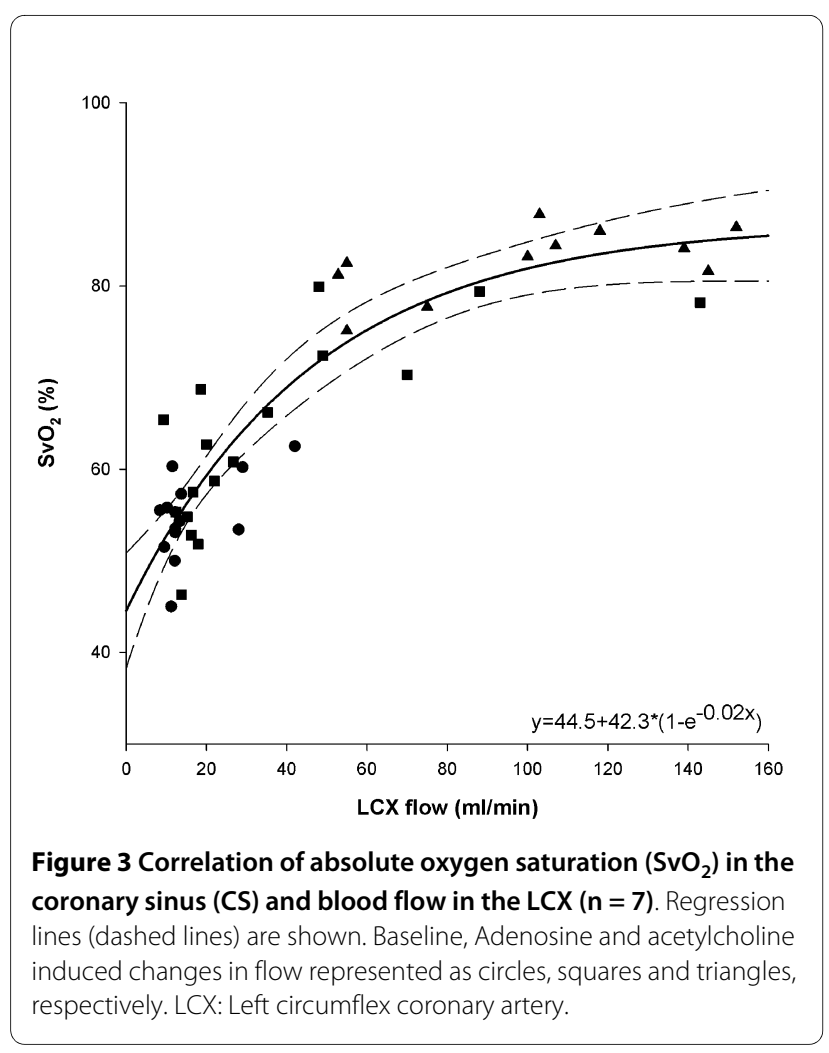

visually apparent (Shown for cine SSFP in Figure 1d). The baseline reproducibility of myocardial BOLD-CMR signal intensity in the LCX perfusion territory was excellent (SEM 0.3\%). $\mathrm{T}_{2}{ }^{*}$ measurements showed an SEM of $6.8 \%$ at baseline and $1.1 \%$ during the protocol.

To see how well CMR tissue measures of oxygenation might predict coronary sinus $\mathrm{SvO}_{2}$, we plotted the change in $\mathrm{SvO}_{2}$ versus myocardial signal intensity (Figure 4) both expressed as percentages, relative to their respective baseline values. Coronary sinus $\mathrm{SvO} 2$ was linearly and strongly correlated with myocardial signal intensity in the SSFP BOLD-CMR images $(\mathrm{r} 2=0.74, \mathrm{P}<0.001)$. T2* changes were relatively larger but showed increased variability $(\mathrm{r} 2=0.34, \mathrm{p}<0.001)$.

\section{Discussion}

Our in vivo study indicates that changes in BOLD CMR signal intensity can be used to predict changes in myocardial oxygenation (as indicated by changes in coronary sinus oxygen saturation) accurately, and with a precision that promises to make the technique clinically useful.

To our knowledge, this is the first in vivo study to compare oxygenation-sensitive CMR using a state-of-the-art SSFP sequence against simultaneous coronary venous blood sampling. Similar studies have been done using $\mathrm{T}_{2}{ }^{*}$ mapping for assessing blood oxygenation[18]. Our results showed an excellent linear correlation of coronary sinus oxygen saturation with CMR signal intensity. $\mathrm{T}_{2}{ }^{*}$ mapping was more sensitive to oxygenation changes but showed more variability. The fact that BOLD CMR shows a linear correlation to coronary sinus oxygen saturation is in agreement with the expected proportionality between the BOLD effect and the absolute tissue content of deoxygenated hemoglobin[13,19,20]. Importantly, a previous theoretical study by Dharmakumar et al. demonstrated that the BOLD signal is blood volume-independent[21].

As shown in Figure 3, the relationship between coronary sinus $\mathrm{SvO}_{2}$ and LCX flow can be described using a 3parameter, exponential-rise equation. With constant myocardial oxygen consumption (i.e., constant RPP), cor-

Table 1: Physiological parameters at each experimental level

\begin{tabular}{|c|c|c|c|c|c|}
\hline & BPsys (mmHg) & HR (min-1) & $\begin{array}{c}\text { RPP }(\mathbf{m m H g} / \\
\text { min) }\end{array}$ & Flow (\%) & SvO2 (\%) \\
\hline BL1 & $95.9 \pm 2.2$ & $72.0 \pm 3.9$ & $6865 \pm 276$ & $100.0 \pm 0$ & $0 \pm 0$ \\
\hline Ach1 & $95.6 \pm 2.5$ & $69.9 \pm 3.2$ & $6650 \pm 258$ & $127.7^{*} \pm 4.6$ & $3.6 \pm 2.3$ \\
\hline Ach2 & $98.3 \pm 1.7$ & $67.3 \pm 3.9$ & $6598 \pm 263$ & $186.2^{*} \pm 13.3$ & $5.3 \pm 2.1$ \\
\hline Ach3 & $99.1 \pm 2.8$ & $65.6 \pm 3.5$ & $6466 \pm 282$ & $309.7^{*} \pm 29.8$ & $15.7^{*} \pm 0.9$ \\
\hline BL2 & $98.5 \pm 3.0$ & $67.2 \pm 3.5$ & $6577 \pm 232$ & $107.5 \pm 5.2$ & $5.2 \pm 2.0$ \\
\hline Ade1 & $97.7 \pm 2.3$ & $73.0 \pm 3.2$ & $7123 \pm 304$ & $817.9^{*} \pm 122.1$ & $31.3^{*} \pm 2.6$ \\
\hline Ade2 & $95.6 \pm 4.1$ & $73.4 \pm 2.7$ & $7018 \pm 410$ & $410.4^{*} \pm 64.7$ & $28.9^{*} \pm 1.2$ \\
\hline Ade3 & $99.0 \pm 1.5$ & $74.0 \pm 8.7$ & $7352 \pm 981$ & $532.2^{*} \pm 56.7$ & $31.5^{*} \pm 0.6$ \\
\hline BL3 & $94.6 \pm 2.2$ & $71.6 \pm 2.3$ & $6769 \pm 259$ & $117.2 \pm 6.4$ & $4.4 \pm 1.4$ \\
\hline
\end{tabular}

$\mathrm{p}<0.05$ compared to baseline

Mean values and SEM for systolic blood pressure (sysBP), heart rate (HR), rate-pressure product (RPP), flow in the left circumflex coronary artery (flow) relative to baseline 1 and change in coronary sinus oxygen saturation $\left(\mathrm{SvO}_{2}\right)$ from baseline 1 at the different experimental levels (BL 1-3: baseline, ACh 1-3: intracoronary acetylcholine infusion/increasing doses, Ade 1-3: intracoronary adenosine infusion/increasing doses; detailed drug doses in the text). ${ }^{*}: p<0.05$ vs. baseline (BL1). 


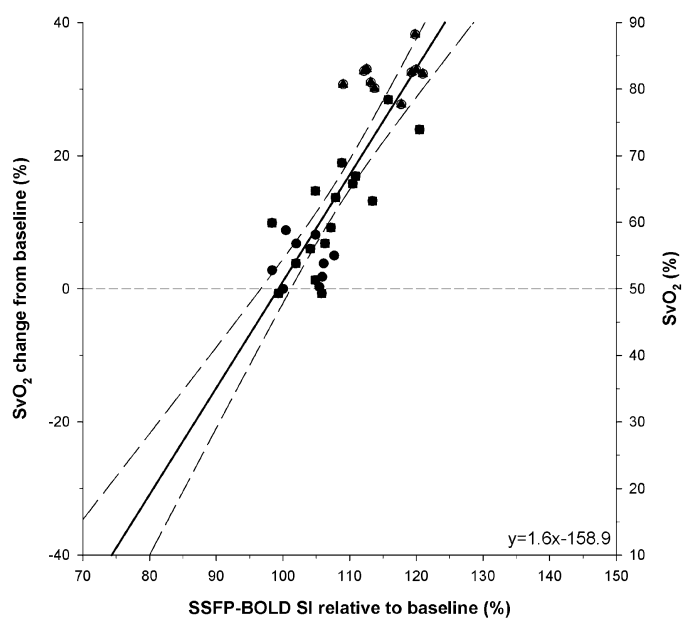

Figure 4 Linear correlation of $\mathrm{SvO}_{2}$ changes from baseline values versus myocardial BOLD-CMR signal intensity relative to baseline $(\mathbf{n}=\mathbf{6})$. Regression lines (dashed lines) are shown. Baseline, adenosine and acetylcholine induced changes in flow represented as circles, squares and triangles, respectively. Figure 4 includes the results of the analyses of extrapolated lines. See text. LCX = left circumflex coronary artery.

onary sinus $\mathrm{SvO}_{2}$ approached an asymptotic value (87\%) as the LCX was over-perfused with near-saturated (97\%) arterial blood under the influence of coronary vasodilators (ACh and Ade).

We chose to use both acetylcholine and adenosine to demonstrate that this technique was sensitive to both endothelium-dependent and -independent vasodilation. The same relationship between myocardial BOLD-CMR signal intensity, coronary blood flow, and $\mathrm{SvO}_{2}$ was observed for ACh- and Ade-induced flow changes (see Figures 3 and 4). This also excludes a significant impact of nitric oxide on myocardial oxygen consumption in acetylcholine-induced vasodilation, as previously speculated[22]. Therefore, SSFP BOLD-CMR appears suitable for studies with both, endothelium-dependent as well as endothelium-independent vasodilation.

In our study, we uncoupled myocardial oxygen consumption from myocardial blood flow by infusing an intracoronary vasodilator that increased blood flow but not heart rate, blood pressure, or RPP (Table 1), which is a surrogate marker for myocardial oxygen consumption[23]. Thus, our model allowed for a selective assessment of perfusion-induced changes while keeping myocardial oxygen consumption stable.

\section{Potential for detection of myocardial ischemia}

Obviously, it would have been of great interest to be able to follow this relationship into the ischemic range but, unfortunately, our attempts to limit LCX flow were not successful in this experimental preparation. However, the linearity and highly correlated nature of the relation between $\mathrm{SvO}_{2}$ and $\mathrm{CMR}$ signal intensity suggest that some extrapolation into the ischemic range would be justified. Based on the control values of LCX flow and the correlations we obtained (see Figure 3), we estimated that the normal average value of coronary sinus $\mathrm{SvO}_{2}$ at baseline was $50 \%$ and that the maximum value was $\sim 90 \%$. Accordingly, we used these values (see right-hand ordinate in Figure 4) to express $\mathrm{SvO}_{2}$ relatively, as the change from this control value in $\% \mathrm{SvO}_{2}$. Thus, we were able to plot changes in coronary sinus $\mathrm{SvO}_{2}$ versus relative value of BOLD CMR signal intensity (Figure 4) throughout the range of LCX flows that we observed and extrapolate into the ischemic ranges (note the extrapolation of the linear regression line). Further experimental and clinical studies should be undertaken to test the accuracy and utility of BOLD-CMR in the presence of myocardial ischemia.

\section{Comparison to Previous Studies}

The effects of ACh and Ade on coronary flow shown in this study are within the range of previously reported studies $[16,17,24]$. Foltz et al. found similar changes in coronary venous oxygen saturation by intracoronary adenosine infusion in the porcine $\mathrm{LAD}[17]$. The corresponding changes in $\mathrm{T}_{2}$ they found during maximal vasodilation were in the range of the $\mathrm{T}_{2}^{*}$ changes we observed, although our $\mathrm{T}_{2}$ * mapping technique did not provide the same consistency. This may be due to the differences in MR techniques and/or differences in coronary territories assessed (LAD vs. LCX). In our protocol, we included the inferolateral wall, which is particularly susceptible for offresonance artifacts in BOLD-sensitive CMR[4]. In a similar experimental approach, Fieno et al. could also demonstrate a close correlation of myocardial perfusion and BOLD-sensitive CMR[11]. They used a different BOLDsensitive CMR technique, which resulted in relatively higher SI changes (up to 30\%), which, however, were linearly correlated to myocardial flow, whereas we found an exponential correlation that matches the $\mathrm{T}_{2}{ }^{*}$ and $\mathrm{SvO}_{2}$ changes. We believe that this difference might be explained by $\mathrm{T}_{2}$ and $\mathrm{T}_{1}$ interference in $\mathrm{T}_{2}$-prepared SSFP imaging since the acquisition follows a $\mathrm{T}_{2}$-preparared SSFP imaging since the acquisition follows a $\mathrm{T}_{2}$-preparation period followed by signal readout during which $\mathrm{T} 1$ recovery is also expected to occur. Finally, in our method, the relatively short breath hold of less than 15 seconds is an advantage.

\section{Clinical Implications}

Besides its apparent utility for clinical research, BOLDCMR appears particularly useful for the clinical assessment of coronary perfusion reserve as used for the detection of relevant coronary artery stenoses. Since BOLD- 
CMR provides a biomarker (Hb oxygenation) instead of surrogate markers, it may overcome many limitations of currently used diagnostic tools. Furthermore, it can be applied as part of a comprehensive CMR study including cardiac morphology, function, coronary anatomy, ischemia, and tissue pathology.

\section{Limitations}

The critical element in our experimental design is that $\mathrm{SvO}_{2}$ should reflect the degree of oxygenation of the LCX myocardium that we evaluated by BOLD SI. We cannot rule out the possibility that there was some admixture by blood draining from areas other than the LCX-perfused myocardium. However, the LCX is the dominant coronary artery in the dog and its course parallels that of the coronary sinus. Furthermore, as pharmacological vasodilatation increased LCX flow, $\mathrm{SvO}_{2}$ approached 87\% -only $10 \%$ below arterial saturation -- suggesting that LCX drainage was overwhelmingly dominant.

\section{Conclusions}

Oxygenation-sensitive CMR using a $\mathrm{T}_{2}{ }^{*}$-sensitive SSFP BOLD sequence allows for non-invasive assessment of changes of myocardial oxygenation in vivo; specifically changes in BOLD signal are impressively proportional to changes in coronary sinus oxygen saturation. Further studies to assess the feasibility and accuracy of this technique in clinical settings are warranted.

\footnotetext{
Abbreviations

BOLD: Blood-Oxygen-Level-Dependent; CMR: Cardiovascular Magnetic Resonance; FOV: Field of view; Fr or F: French; GRE-EPI: GRadient Echo-Echo Planar Imaging; LCX: Left circumflex coronary artery; MRI: Magnetic resonance imaging; Px: Pixel; SSFP: Steady-state-free-precession; $\mathrm{SvO}_{2}$ : Venous oxygen saturation

\section{Competing interests}

The research was supported by the Husky Energy Research Program for the Early Detection of Cardiovascular Risk. During the experiments, Matthias Vöhringer was supported by a research scholarship from Robert-Bosch-Foundation, Germany and was a Canadian Institute of Health Research (CIHR) strategic training fellow in TORCH (Tomorrows Research in Cardiovascular Health Professionals), Rohan Dharmakumar was supported by American Heart Association (SDG 0735099N) and National Institutes of Health (HL 091989), and Jordin Green was a full-time employee with Siemens Canada Limited; Matthias Friedrich is a scientific advisor and stockholder of Circle Cardiovascular Imaging Inc.
}

\section{Authors' contributions}

MV has performed the invasive experiments, collected the data, performed the evaluation and was significantly involved in writing the manuscript. JAF was responsible for the experimental setup of the experiments and in gathering and documenting the data. She participated in the scanning procedures and was significantly involved in writing the manuscript. JDG has modified the CMR sequences for our study. He was significantly involved in all scanning procedures and in the preparation of the manuscript. RD developed the CMR sequence and was involved in designing the CMR experiments and in writing the manuscript. JJW was involved in evaluation of the data related to analyses of coronary blood flow and significantly involved in preparing the figures. JVT was significantly involved in designing the experiments and the protocol and in writing the manuscript. MGF was responsible for the overall concept and was significantly involved in the study and protocol design. He was also significantly involved in writing the manuscript.

\section{Acknowledgements}

We cordially thank Cheryl Meek for her excellent animal preparation and technical assistance.

\section{Author Details}

1Stephenson Cardiovascular MR Centre at the Libin Cardiovascular Institute of Alberta, Department of Cardiac Sciences, University of Calgary, Calgary, AB, Canada, ${ }^{2}$ Robert-Bosch-Krankenhaus, Stuttgart, Germany, ${ }^{3}$ Siemens Healthcare, Erlangen, Germany, ${ }^{4}$ Department of Radiology, Northwestern University, Chicago, IL, USA, ${ }^{5}$ Fu-Jen University Medical School, Hsinchuang, Taiwan, ${ }^{6}$ Department of Cardiac Sciences and Physiology/Pharmacology, University of Calgary, Calgary, AB, Canada and 7Stephenson Cardiovascular MR Centre at the Libin Cardiovascular Institute of Alberta, Department of Cardiac Sciences, University of Calgary, Calgary, AB, Canada

Received: 1 October 2009 Accepted: 31 March 2010 Published: 31 March 2010

\section{References}

1. Feigl EO: Coronary physiology. Physiol Rev 1983, 63:1-205.

2. Wilke NM, Jerosch-Herold M, Zenovich A, Stillman AE: Magnetic resonance first-pass myocardial perfusion imaging: clinical validation and future applications. J Magn Reson Imaging 1999, 10:676-685.

3. McCommis KS, Goldstein TA, Abendschein DR, Herrero P, Misselwitz B, Gropler RJ, Zheng J: Quantification of regional myocardial oxygenation by magnetic resonance imaging: validation with positron emission tomography. Circ Cardiovasc Imaging 2010, 3:41-46.

4. Kennan RP, Zhong J, Gore JC: Intravascular susceptibility contrast mechanisms in tissues. Magn Reson Med 1994, 31:9-21.

5. Wendland MF, Saeed M, Lauerma K, de Crespigny A, Moseley ME, Higgins CB: Endogenous susceptibility contrast in myocardium during apnea measured using gradient recalled echo planar imaging. Magn Reson Med 1993, 29:273-276.

6. Ogawa S, Tank DW, Menon R, Ellermann JM, Kim SG, Merkle H, Ugurbil K: Intrinsic signal changes accompanying sensory stimulation: functional brain mapping with magnetic resonance imaging. Proc Natl Acad Sci USA 1992, 89:5951-5955

7. Wacker CM, Bock M, Hartlep AW, Bauer WR, van Kaick G, Pfleger S, Ertl G, Schad LR: BOLD-MRI in ten patients with coronary artery disease: evidence for imaging of capillary recruitment in myocardium supplied by the stenotic artery. MAGMA 1999, 8:48-54.

8. Utz W, Jordan J, Niendorf T, Stoffels M, Luft FC, Dietz R, Friedrich M: Blood oxygen level-dependent MRI of tissue oxygenation: relation to endothelium-dependent and endothelium-independent blood flow changes. Arterioscler Thromb Vasc Biol 2005, 25:1408-1413.

9. Friedrich M, Niendorf T, Schulz-Menger J, Gross CM, Dietz R: Blood oxygen level-dependent magnetic resonance imaging in patients with stress-induced angina. Circulation 2003, 108:2219-2223.

10. Li D, Dhawale P, Rubin PJ, Haacke EM, Gropler RJ: Myocardial signal response to dipyridamole and dobutamine: demonstration of the BOLD effect using a double-echo gradient-echo sequence. Magn Reson Med 1996, 36:16-20.

11. Fieno DS, Shea SM, Li Y, Harris KR, Finn JP, Li D: Myocardial perfusion imaging based on the blood oxygen level-dependent effect using T2 prepared steady-state free-precession magnetic resonance imaging. Circulation 2004, 110:1284-1290.

12. Arumana JM, Li D, Dharmakumar R: Deriving blood-oxygen-leveldependent contrast in MRI with T2*-weighted, T2-prepared and phase-cycled SSFP methods: theory and experiment. Magn Reson Med 2008, 59:561-570

13. Dharmakumar R, Hong J, Brittain JH, Plewes DB, Wright GA: Oxygensensitive contrast in blood for steady-state free precession imaging. Magn Reson Med 2005, 53:574-583.

14. Dharmakumar R, Arumana JM, Tang R, Harris K, Zhang Z, Li D: Assessment of regional myocardial oxygenation changes in the presence of coronary artery stenosis with balanced SSFP imaging at 3.0 T: theory and experimental evaluation in canines. J Magn Reson Imaging 2008, 27:1037-1045.

15. Dharmakumar R, Arumana JM, Larson AC, Chung Y, Wright GA, Li D: Cardiac phase-resolved blood oxygen-sensitive steady-state free precession MRI for evaluating the functional significance of coronary artery stenosis. Invest Radiol 2007, 42:180-188. 
16. Gill RM, Braz JC, Jin N, Etgen GJ, Shen W: Restoration of impaired endothelium-dependent coronary vasodilation in failing heart: role of eNOS phosphorylation and CGMP/CGK-I signaling. Am J Physiol Heart Circ Physiol 2007, 292:H2782-2790

17. Foltz WD, Huang H, Fort $\mathrm{S}$, Wright GA: Vasodilator response assessment in porcine myocardium with magnetic resonance relaxometry. Circulation 2002, 106:2714-2719.

18. Li D, Waight DJ, Wang Y: In vivo correlation between blood T2* and oxygen saturation. J Magn Reson Imaging 1998, 8:1236-1239.

19. Ogawa S, Menon RS, Tank DW, Kim SG, Merkle H, Ellermann JM, Ugurbil K: Functional brain mapping by blood oxygenation level-dependent contrast magnetic resonance imaging. A comparison of signal characteristics with a biophysical model. Biophys J 1993, 64:803-812.

20. Wacker CM, Bock M, Hartlep AW, Beck G, van Kaick G, Ertl G, Bauer WR, Schad LR: Changes in myocardial oxygenation and perfusion under pharmacological stress with dipyridamole: assessment using $T^{*} 2$ and T1 measurements. Magn Reson Med 1999, 41:686-695.

21. Dharmakumar R, Qi X, Hong J, Wright GA: Detecting microcirculatory changes in blood oxygen state with steady-state free precession imaging. Magn Reson Med 2006, 55:1372-1380.

22. Trochu JN, Bouhour JB, Kaley G, Hintze TH: Role of endothelium-derived nitric oxide in the regulation of cardiac oxygen metabolism: implications in health and disease. Circ Res 2000, 87:1108-1117.

23. Gobel FL, Norstrom LA, Nelson RR, Jorgensen CR, Wang Y: The ratepressure product as an index of myocardial oxygen consumption during exercise in patients with angina pectoris. Circulation 1978, 57:549-556.

24. Dick GM, Katz PS, Farias M, Morris M, James J, Knudson JD, Tune JD: Resistin impairs endothelium-dependent dilation to bradykinin, but not acetylcholine, in the coronary circulation. Am J Physiol Heart Circ Physiol 2006, 291:H2997-3002.

doi: $10.1186 / 1532-429 X-12-20$

Cite this article as: Vöhringer et al., Oxygenation-sensitive CMR for assessing vasodilator-induced changes of myocardial oxygenation Journal of Cardiovascular Magnetic Resonance 2010, 12:20

Submit your next manuscript to BioMed Central and take full advantage of:

- Convenient online submission

- Thorough peer review

- No space constraints or color figure charges

- Immediate publication on acceptance

- Inclusion in PubMed, CAS, Scopus and Google Scholar

- Research which is freely available for redistribution

Submit your manuscript at www.biomedcentral.com/submit
C) BioMed Central 